\title{
MOORE'S CONJECTURE FOR POLYHEDRAL PRODUCTS
}

\author{
YANLONG HAO*, QIANWEN SUN*, AND STEPHEN THERIAULT
}

Abstract. Moore's Conjecture is shown to hold for generalized moment-angle complexes and a criterion is proved that determines when a polyhedral product is elliptic or hyperbolic.

\section{INTRODUCTION}

Moore's Conjecture envisions a deep relationship between the rational and torsion homotopy groups of finite $C W$-complexes. Let $X$ be a finite $C W$-complex. The homotopy exponent of $X$ at a prime $p$ is the least power of $p$ that annihilates the $p$-torsion in the homotopy groups of $X$. The space $X$ is elliptic if it has finitely many rational homotopy groups and hyperbolic if it has infinitely many rational homotopy groups.

Moore's Conjecture: Let $X$ be a finite, simply-connected $C W$-complex. Then the following are equivalent:

(a) $X$ is elliptic;

(b) $X$ has a finite homotopy exponent at every prime $p$;

(c) $X$ has a finite homotopy exponent at some prime $p$.

The conjecture posits that the nature of the rational homotopy groups should have a profound impact on the nature of the, seemingly unrelated, torsion homotopy groups, and that torsion behaviour at one prime has a profound impact on torsion behaviour at all primes. The conjecture has been shown to hold in a number of cases. Elliptic spaces with finite exponents at all primes include spheres [13, 22], finite $H$-spaces [14], $H$-spaces with finitely generated cohomology [5], and odd primary Moore spaces [17]. Hyperbolic spaces with no exponent at any prime include wedges of simply-connected spheres, most torsion-free two-cell complexes [18], and torsion-free suspensions [20]. There are also partial results. In [16] it was shown that if $X$ is elliptic then it has an exponent at all but finitely many primes, in [21] it was shown that if $X$ is hyperbolic and $H_{*}(\Omega X ; \mathbb{Z})$ is $p$-torsion free then, provided $p$ is large enough, $X$ has no exponent at $p$, and in [1] Moore's Conjecture was shown to hold for all but finitely many primes in the case of spaces having Lusternik-Schnirelmann category two.

2010 Mathematics Subject Classification. Primary 55Q05, Secondary 13F55, 55P62, 55U10.

Key words and phrases. Moore's conjecture, elliptic, hyperbolic, polyhedral product.

* Research supported by the National Natural Science Foundation of China (No. 11571186). 
Moore's conjecture is also related to an important phenomenon in rational homotopy theory. Félix, Halperin and Thomas [8] proved the remarkable fact that a finite $C W$-complex is either elliptic or its total number of rational homotopy groups below dimension $n$ grows exponentially with $n$. There is no hyperbolic space whose rational homotopy groups have polynomial growth.

In this paper we consider Moore's conjecture, and the notions of being elliptic or hyperbolic, in the context of polyhedral products. Let $K$ be a simplicial complex on $m$ vertices. For $1 \leq i \leq m$, let $\left(X_{i}, A_{i}\right)$ be a pair of pointed $C W$-complexes, where $A_{i}$ is a pointed subspace of $X_{i}$. Let $(\underline{X}, \underline{A})=$ $\left\{\left(X_{i}, A_{i}\right)\right\}_{i=1}^{m}$ be the sequence of $C W$-pairs. For each simplex (face) $\sigma \in K$, let $(\underline{X}, \underline{A})^{\sigma}$ be the subspace of $\prod_{i=1}^{m} X_{i}$ defined by

$$
(\underline{X}, \underline{A})^{\sigma}=\prod_{i=1}^{m} \bar{X}_{i} \quad \text { where } \quad \bar{X}_{i}=\left\{\begin{array}{cl}
X_{i} & \text { if } i \in \sigma \\
A_{i} & \text { if } i \notin \sigma .
\end{array}\right.
$$

The polyhedral product determined by $(\underline{X}, \underline{A})$ and $K$ is

$$
(\underline{X}, \underline{A})^{K}=\bigcup_{\sigma \in K}(\underline{X}, \underline{A})^{\sigma} \subseteq \prod_{i=1}^{m} X_{i} .
$$

The topology of polyhedral products has received a great deal of attention recently due to their central role in toric topology $[2,4,10,11,12]$. Important special cases include moment-angle complexes $\mathcal{Z}_{K}$, when each pair $\left(X_{i}, A_{i}\right)$ equals $\left(D^{2}, S^{1}\right)$, and generalized moment-angle complexes $\mathcal{Z}_{K}\left(D^{n}, S^{n-1}\right)$, when each pair $\left(X_{i}, A_{i}\right)$ equals $\left(D^{n}, S^{n-1}\right)$ for $n \geq 2$. By [4], each generalized moment-angle complex is a finite, simply-connected $C W$-complex, so Moore's Conjecture may be considered.

To state our results some definitions are needed. Write $[m]$ for the vertex set $\{1, \ldots, m\}$. Let $\Delta^{m-1}$ be the standard $m$-simplex with vertex set $[m]$. The faces of $\Delta^{m-1}$ can be identified with sequences $\left(i_{1}, \ldots, i_{k}\right)$ for $1 \leq i_{1}<\cdots<i_{k} \leq m$. If $K$ is a simplicial complex on the vertex set $[m]$ then a sequence $\sigma=\left(i_{1}, \ldots, i_{k}\right)$ is a missing face of $K$ if $\sigma \notin K$. It is a minimal missing face of $K$ if no proper subsequence of $\sigma$ is a missing face of $K$.

Theorem 1.1. Let $K$ be a simplicial complex on the vertex set $[m]$ and let $(\underline{X}, \underline{A})$ be any sequence of pairs $\left(D^{n_{i}}, S^{n_{i}-1}\right)$ with $n_{i} \geq 2$ for $1 \leq i \leq m$. Then:

(a) $(\underline{X}, \underline{A})^{K}$ is elliptic if and only if the minimal missing faces of $K$ are mutually disjoint;

(b) Moore's conjecture holds for $(\underline{X}, \underline{A})^{K}$.

In particular, Theorem 1.1 includes generalized moment-angle complexes $\mathcal{Z}_{K}\left(D^{n}, S^{n-1}\right)$ for $n \geq 2$ as a special case. Part (a) of Theorem 1.1 was proved by [2] in the special case of the momentangle complex $\mathcal{Z}_{K}$, although part (b) was not. The restriction to $n \geq 2$ is made to ensure that certain retractions constructed in Theorem 4.2 involve wedges of simply-connected spheres which are hyperbolic, rather than wedge of circles which are Eilenberg-MacLane spaces. 
We also give a general criterion for when a polyhedral product is elliptic or hyperbolic. This generalizes and reformulates in more combinatorial terms results obtained by Félix and Tanré [9].

Theorem 1.2. Let $K$ be a simplicial complex on the vertex set $[m]$ and let $(\underline{X}, \underline{A})$ be any sequence of pointed, path-connected pairs. For $1 \leq i \leq m$, let $Y_{i}$ be the homotopy fibre of the inclusion $A_{i} \longrightarrow X_{i}$ and suppose that each $Y_{i}$ is rationally nontrivial. Then the polyhedral product $(\underline{X}, \underline{A})^{K}$ is elliptic if and only if three conditions hold:

(i) each $X_{i}$ is elliptic;

(ii) all the minimal missing faces of $K$ are mutually disjoint;

(iii) if $v$ is a vertex of a minimal missing face of $K$ then $Y_{v}$ is rationally homotopy equivalent to a sphere.

For example, let $K$ be the boundary of a pentagon. A result essentially due to MacGavran [15] shows that $\mathcal{Z}_{K}$ is diffeomorphic to the connected sum of 5 copies of $S^{3} \times S^{4}$. It is well known that such a connected sum is hyperbolic. If the ingredient pairs of spaces change from $\left(D^{2}, S^{1}\right)$ to $\left(C \mathbb{C} P^{n}, \mathbb{C} P^{n}\right)$, where $C \mathbb{C} P^{n}$ is the cone on $\mathbb{C} P^{n}$, then the polyhedral product $\left(\underline{C \mathbb{C} P^{n}}, \underline{\mathbb{C} P^{n}}\right)^{K}$ is some analogue of a connected sum, but its homotopy type is not clear. Observe that each $Y_{i}$ in this case is $\Omega \mathbb{C} P^{n}$, so is rationally nontrivial, and the minimal missing faces of $K$ are not mutually disjoint. So Theorem 1.2 implies that $\left(\underline{C \mathbb{C} P^{n}}, \underline{\mathbb{C} P^{n}}\right)^{K}$ is hyperbolic. The most pertinent point here is that this determination is made without any reference to the standard differential graded Lie algebra tools commonly used to decide ellipticity or hyperbolicity.

The authors would like to thank the referee for suggestions that improved the clarity of the paper.

\section{Polyhedral Product ingredients}

This section contains the properties of polyhedral products that will be needed. The main results are Theorems 2.1 and 2.3, which are of independent interest.

Theorem 2.1. Let $K$ be a simplicial complex on the vertex set $[m]$ and let $(\underline{X}, \underline{A})$ be any sequence of pointed, path-connected $C W$-pairs. Then there is a homotopy fibration

$$
(\underline{C Y}, \underline{Y})^{K} \longrightarrow(\underline{X}, \underline{A})^{K} \longrightarrow \prod_{i=1}^{m} X_{i}
$$

where, for $1 \leq i \leq m, Y_{i}$ is the homotopy fibre of the inclusion $A_{i} \longrightarrow X_{i}$.

Proof. In general, let $f: B \longrightarrow Z$ be a pointed, continuous map, let $I=[0,1]$ be the unit interval with basepoint 0 , and let $P Z$ be the path space of $Z$. Then the homotopy fibre of $f$ is the pullback of $f$ and the evaluation map $e v_{1}: P Z \longrightarrow Z$, where $e v_{1}(\omega)=\omega(1)$. In our case, we are given $m$ pairs of spaces $\left(X_{i}, A_{i}\right)$. The homotopy pullback of the identity map $1_{X_{i}}: X_{i} \longrightarrow X_{i}$ is $P X_{i}$, and $Y_{i}$ is defined as the homotopy pullback of the inclusion $j_{i}: A_{i} \longrightarrow X_{i}$. Note that as $j_{i}$ is a subspace inclusion then $Y_{i}$ is the inverse image $e v_{1}^{-1}\left(A_{i}\right) \subseteq P X_{i}$. 
Let $\sigma$ be a face in $K$. By the definition of a polyhedral product, $(\underline{X}, \underline{A})^{\sigma}=\bar{X}_{1} \times \cdots \times \bar{X}_{m}$, where $\bar{X}_{i}$ is $X_{i}$ if $i \in \sigma$ and is $A_{i}$ if $i \notin \sigma$. Consider the homotopy pullback

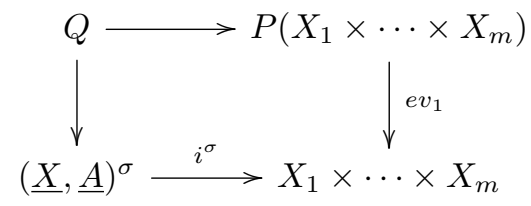

where $i^{\sigma}$ is the inclusion. Observe that $P\left(X_{1} \times \cdots \times \cdots X_{m}\right)$ is homeomorphic to $P X_{1} \times \cdots \times P X_{m}$ and under this homeomorphism $e v_{1}$ translates into a product of the $m$ evaluation maps $e v_{1}$ on each $P X_{i}$. As the product of pullbacks is a pullback, we see that $Q$ is homeomorphic to $\bar{Y}_{1} \times \cdots \times \bar{Y}_{m}$, where $\bar{Y}_{i}$ is $P X_{i}$ if $i \in \sigma$ and is $Y_{i}$ if $i \notin \sigma$. That is, $Q$ is homeomorphic to $(\underline{P X}, \underline{Y})^{\sigma}$. Moreover, since $i^{\sigma}$ is an inclusion, $(\underline{P X}, \underline{Y})^{\sigma}$ is the inverse image $\left(e v_{1} \times \cdots \times e v_{1}\right)^{-1}\left((\underline{X}, \underline{A})^{\sigma}\right) \subseteq P X_{1} \times \cdots \times P X_{m}$. Now $(\underline{X}, \underline{A})^{K}$ is the union of the spaces $(\underline{X}, \underline{A})^{\sigma}$ for all $\sigma \in K$, where intersections have been identified. Since inverse images preserve unions and intersections, we obtain that the homotopy fibre of the inclusion $(\underline{X}, \underline{A})^{K} \longrightarrow X_{1} \times \cdots \times X_{m}$ is homeomorphic to $(\underline{P X}, \underline{Y})^{K}$.

Finally, since $P X_{i}$ is contractible, the inclusion $Y_{i} \longrightarrow P X_{i}$ extends to a map $C Y_{i} \longrightarrow P X_{i}$ which is a homotopy equivalence. Thus the induced map of pairs $\left(C Y_{i}, Y_{i}\right) \longrightarrow\left(P X_{i}, Y_{i}\right)$ is a homotopy equivalence. Hence the homotopy fibre of the inclusion $(\underline{X}, \underline{A})^{K} \longrightarrow X_{1} \times \cdots \times X_{m}$ is homotopy equivalent to $(\underline{C Y}, \underline{Y})^{K}$

Next, we show that the homotopy fibration in Theorem 2.1 splits after looping. Let $K$ be a simplicial complex on the vertex set $[m]$. If $I \subseteq[m]$ then the full subcomplex $K_{I}$ of $K$ is defined as the simplicial complex

$$
K_{I}=\bigcup\{\sigma \in K \mid \text { the vertex set of } \sigma \text { is in } I\} .
$$

The definition of $K_{I}$ implies that the inclusion $K_{I} \longrightarrow K$ is a map of simplicial complexes. This induces a map of polyhedral products $(\underline{X}, \underline{A})^{K_{I}} \longrightarrow(\underline{X}, \underline{A})^{K}$. There is no retraction of $K_{I}$ off $K$ as simplicial complexes, however, in [7] it was shown that there is nevertheless a retraction of $(\underline{X}, \underline{A})^{K_{I}}$ off $(\underline{X}, \underline{A})^{K}$.

Proposition 2.2. Let $K$ be a simplicial complex on the vertex set $[m]$ and let $(\underline{X}, \underline{A})$ be any sequence of pointed, path-connected $C W$-pairs. Let $I \subseteq[m]$. Then the inclusion $(\underline{X}, \underline{A})^{K_{I}} \longrightarrow(\underline{X}, \underline{A})^{K}$ has a left inverse.

Theorem 2.3. Let $(\underline{C Y}, \underline{Y})^{K} \longrightarrow(\underline{X}, \underline{A})^{K} \longrightarrow \prod_{i=1}^{m} X_{i}$ be the homotopy fibration in Theorem 2.1. Then there is a homotopy equivalence

$$
\Omega(\underline{X}, \underline{A})^{K} \simeq\left(\prod_{i=1}^{m} \Omega X_{i}\right) \times \Omega(\underline{C Y}, \underline{Y})^{K} .
$$

Proof. For $1 \leq i \leq m$, let $I_{i}=\{i\}$. Observe that the full subcomplex $K_{I_{i}}$ of $K$ is just the vertex $\{i\}$. By the definition of the polyhedral product, $(\underline{X}, \underline{A})^{K_{I_{i}}}=X_{i}$. Proposition 2.2 therefore 
implies that $X_{i}$ retracts off $(\underline{X}, \underline{A})^{K}$. Explicitly, the composite $X_{i}=(\underline{X}, \underline{A})^{K_{I_{i}}} \longrightarrow(\underline{X}, \underline{A})^{K} \longrightarrow$ $\prod_{i=1}^{m} X_{i} \stackrel{\text { proj }}{\longrightarrow} X_{i}$ is the identity map. After looping, the loop maps $\Omega X_{i} \longrightarrow \Omega(\underline{X}, \underline{A})^{K}$ may be multiplied together to obtain a map $\prod_{i=1}^{m} \Omega X_{i} \longrightarrow \Omega(\underline{X}, \underline{A})^{K}$ which is a right homotopy inverse of the map $\Omega(\underline{X}, \underline{A})^{K} \longrightarrow \prod_{i=1}^{m} \Omega X_{i}$. Hence, if $\mu$ is the loop multiplication on $\Omega(\underline{X}, \underline{A})^{K}$, then the composite

$$
\left(\prod_{i=1}^{m} \Omega X_{i}\right) \times \Omega(\underline{C Y}, \underline{Y})^{K} \longrightarrow \Omega(\underline{X}, \underline{A})^{K} \times \Omega(\underline{X}, \underline{A})^{K} \stackrel{\mu}{\longrightarrow} \Omega(\underline{X}, \underline{A})^{K}
$$

is a homotopy equivalence.

Theorem 2.3 implies that homotopy group information about $(\underline{X}, \underline{A})^{K}$ is determined by that of the ingredient spaces $X_{i}$ and $(\underline{C Y}, \underline{Y})^{K}$. This is useful because the spaces $(\underline{C Y}, \underline{Y})^{K}$ are much better understood than the spaces $(\underline{X}, \underline{A})^{K}$.

This section concludes with the statement of two other results which will be used later. The first, proved in [11], relates pushouts of simplicial complexes to pushouts of polyhedral products.

Proposition 2.4. Let $K$ be a simplicial complex on the vertex set $[m]$. Suppose that there is a pushout of simplicial complexes

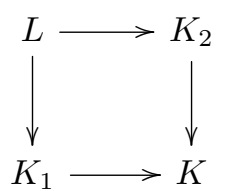

Let $L^{\circ}, K_{1}^{\circ}$ and $K_{1}^{\circ}$ be $L, K_{1}$ and $K_{2}$ regarded as simplicial complexes on the same vertex set as $K$.

Then there is a pushout of polyhedral products

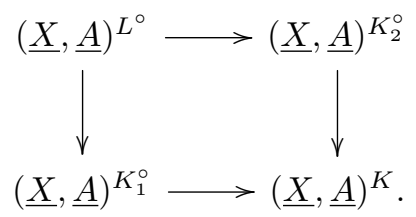

Second, we give two examples where the homotopy type of $(\underline{C Y}, \underline{Y})^{K}$ is explicitly identified. Part (a) in Lemma 2.5 is immediate from the definition of the polyhedral product, while part (b) was proved by Porter [19] when each $Y_{i}$ is a loop space and more generally in [11].

Lemma 2.5. Let $Y_{1}, \ldots, Y_{m}$ be path-connected spaces. Then the following hold:

(a) $(\underline{C Y}, \underline{Y})^{\Delta^{m-1}}=\prod_{i=1}^{m} C Y_{i}$;

(b) $(\underline{C Y}, \underline{Y})^{\partial \Delta^{m-1}} \simeq \Sigma^{m-1} Y_{1} \wedge \cdots \wedge Y_{m}$. 


\section{Combinatorial ingredients}

This section records the combinatorial information that will be needed. Let $K$ be a simplicial complex on the index set $[m]$. For a vertex $v \in K$, the star, restriction (or deletion) and link of $v$ are the subcomplexes

$$
\begin{aligned}
\operatorname{star}_{K}(v) & =\{\tau \in K \mid\{v\} \cup \tau \in K\} ; \\
K \backslash v & =\{\tau \in K \mid\{v\} \cap \tau=\emptyset\} ; \\
\operatorname{link}_{K}(v) & =\operatorname{star}_{K}(v) \cap K \backslash v .
\end{aligned}
$$

The join of two simplicial complexes $K_{1}, K_{2}$ on disjoint index sets is the simplicial complex

$$
K_{1} * K_{2}=\left\{\sigma_{1} \cup \sigma_{2} \mid \sigma_{i} \in K_{i}\right\} .
$$

From the definitions, it follows that $\operatorname{star}_{K}(v)$ is a join,

$$
\operatorname{star}_{K}(v)=\{v\} * \operatorname{link}_{K}(v),
$$

and there is a pushout

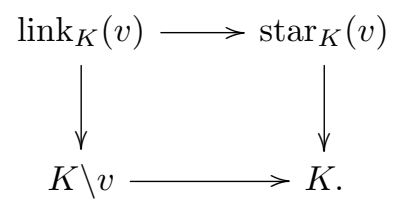

A face of $K$ is a simplex of $K$. Let $\Delta^{m-1}$ be the standard $m$-simplex on the vertex set $[m]$ and note that $K$ is a subcomplex of $\Delta^{m-1}$. Recall from the Introduction that a face $\sigma \in \Delta^{m-1}$ is a missing face of $K$ if $\sigma \notin K$. It is a minimal missing face if any proper face of $\sigma$ is a face of $K$. Denote the set of minimal missing faces of $K$ by $M M F(K)$. For a simplex $\sigma$, let $\partial \sigma$ be its boundary. Observe that $\sigma \in M M F(K)$ if and only if $\sigma \notin K$ but $\partial \sigma \subseteq K$.

There is a special case which will play a crucial role in what follows. Let $\bar{K}$ be a simplicial complex on the vertex set $[m]$ with the property that it has precisely two distinct minimal missing faces and these have non-empty intersection. That is, suppose that $M M F(\bar{K})=\left\{\sigma_{1}, \sigma_{2}\right\}$ where $\sigma_{1}$ and $\sigma_{2}$ have vertex sets $I$ and $J$ respectively, satisfying $I \neq J, I \cup J=[m]$ and $I \cap J \neq \emptyset$. Let $w$ be a vertex in both $I$ and $J$.

Consider the star-link-restriction pushout of $\bar{K}$ with respect to the vertex $w$ :

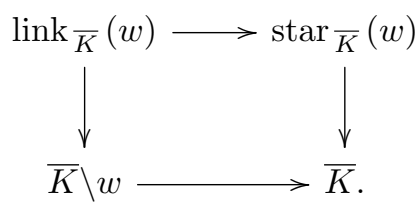

Let $\bar{\sigma}_{1}$ and $\bar{\sigma}_{2}$ be the proper faces of $\sigma_{1}$ and $\sigma_{2}$ on the vertex sets $\bar{I}=I \backslash\{w\}$ and $\bar{J}=J \backslash\{w\}$ respectively.

Lemma 3.1. We have $\bar{\sigma}_{1}, \bar{\sigma}_{2} \in M M F\left(\operatorname{star}_{\bar{K}}(w)\right)$. 
Proof. Consider $\bar{\sigma}_{1}$, the argument for $\bar{\sigma}_{2}$ being similar. First we show that $\bar{\sigma}_{1}$ is a missing face of $\operatorname{star}_{\bar{K}}(w)$. For if $\bar{\sigma}_{1} \in \operatorname{star}_{\bar{K}}(w)$ then, as $w$ is not a vertex of $\bar{\sigma}_{1}$, we also have $\bar{\sigma}_{1} \in \bar{K} \backslash w$, implying that $\bar{\sigma}_{1} \in \operatorname{link}_{\bar{K}}(w)=\operatorname{star}_{\bar{K}}(w) \cap \bar{K} \backslash w$. This in turn implies that $\bar{\sigma}_{1} *\{w\} \in \operatorname{star}_{\bar{K}}(w)$. But $\bar{\sigma}_{1} *\{w\}=\sigma_{1}$, so $\sigma_{1} \in \operatorname{star}_{\bar{K}}(w)$. Therefore, by $(1), \sigma_{1} \in \bar{K}$, contradicting the fact that $\sigma_{1}$ is a missing face of $\bar{K}$.

Next, we show that that $\bar{\sigma}_{1}$ is a minimal missing face of $\operatorname{star}_{\bar{K}}(w)$. If not, then some proper face $\tau$ of $\bar{\sigma}_{1}$ is also a missing face of $\operatorname{star}_{\bar{K}}(w)$. As $w$ is not a vertex of $\bar{\sigma}_{1}$, it is not a vertex of $\tau$ either. Therefore $\tau *\{w\}$ is a missing face of $\operatorname{star}_{\bar{K}}(w)$. The presence of the vertex $w$ in $\tau *\{w\}$ implies that it is also not a face of $\bar{K} \backslash w$. On the other hand, by (1), $\bar{K}$ is the union of $\operatorname{star}_{\bar{K}}(w)$ and $\bar{K} \backslash w$, so a face that is missing from both $\operatorname{star}_{\bar{K}}(w)$ and $\bar{K} \backslash w$ must also be missing from $\bar{K}$. Therefore $\tau *\{w\}$ is a missing face of $\bar{K}$. But as $\tau$ is a proper face of $\bar{\sigma}_{1}, \tau *\{w\}$ is a proper face of $\bar{\sigma}_{1} *\{w\}=\sigma_{1}$, contradicting the fact that $\sigma_{1}$ is a minimial missing face of $\bar{K}$.

Corollary 3.2. We have $\partial \bar{\sigma}_{1}, \partial \bar{\sigma}_{2} \subseteq \operatorname{link}_{\bar{K}}(w)$ and $\bar{\sigma}_{1}, \bar{\sigma}_{2} \notin \operatorname{link} \bar{K}_{\bar{K}}(w)$.

Proof. Recall that a face $\sigma$ of a simplicial complex $K$ is a minimal missing face if and only if $\sigma \notin K$ but $\partial \sigma \subseteq K$. So by Lemma 3.1, $\partial \bar{\sigma}_{1}, \partial \bar{\sigma}_{2} \subseteq \operatorname{star}_{\bar{K}}(w)$. By definition, neither $\bar{\sigma}_{1}$ nor $\bar{\sigma}_{2}$ have $w$ in their vertex sets, so neither do their boundaries. Therefore $\partial \bar{\sigma}_{1}, \partial \bar{\sigma}_{2} \subseteq \bar{K} \backslash w$. Therefore, as $\operatorname{link}_{\bar{K}}(w)=\operatorname{star}_{\bar{K}}(w) \cap \bar{K} \backslash w$, we have $\partial \bar{\sigma}_{1}, \partial \bar{\sigma}_{2} \subseteq \operatorname{link}_{\bar{K}}(w)$.

Also, as $\operatorname{link}_{\bar{K}}(w)=\operatorname{star}_{\bar{K}}(w) \cap \bar{K} \backslash w$, it cannot be that $\bar{\sigma}_{1}, \bar{\sigma}_{2}$ are in $\operatorname{link}_{\bar{K}}(w)$ as that would imply they are also in $\operatorname{star}_{\bar{K}}(w)$, contradicting Lemma 3.1 .

One further observation we need regarding $\bar{K}$ is the following. Regarding $w$ as the $m^{\text {th }}$-vertex of $\bar{K}$, observe that $\bar{K} \backslash w$ is a simplicial complex on the vertex set $[m-1]$.

Lemma 3.3. There is an isomorphism of simplicial complexes $\bar{K} \backslash w \cong \Delta^{m-2}$.

Proof. It is equivalent to show that $\bar{K} \backslash w$ has no missing faces. Suppose that $\sigma \in \Delta^{m-2}$ is a missing face of $\bar{K} \backslash w$. Then as $\bar{K} \backslash w$ is the restriction of $\bar{K}$ to the vertex set [m-1], $\sigma$ is also a missing face of $\bar{K}$. On the other hand, as $M M F(\bar{K})=\left\{\sigma_{1}, \sigma_{2}\right\}$, any missing face of $\bar{K}$ must have either $\sigma_{1}$ or $\sigma_{2}$ as a subface. Thus $\sigma$ must have either $\sigma_{1}$ or $\sigma_{2}$ as a subface. But this cannot happen since $w$ is not in the vertex set of $\sigma$ but it is in the vertex sets of both $\sigma_{1}$ and $\sigma_{2}$.

\section{Moore's CONJECTURE}

In this section we prove Theorems 1.1 and 1.2 as consequences of Theorem 4.2.

Proposition 4.1. Let $K$ be a simplicial complex on the vertex set $[m]$ and let $X_{1}, \ldots, X_{m}$ be any sequence of pointed, path-connected $C W$-pairs. Suppose that $\sigma_{1}, \sigma_{2} \in M M F(K)$ and let $I$ and $J$ be the vertex sets of $\sigma_{1}$ and $\sigma_{2}$ respectively. If $I \neq J, I \cup J=[m]$ and $I \cap J \neq \emptyset$, then $(\underline{C X}, \underline{X})^{\partial \sigma_{1}} \vee(\underline{C X}, \underline{X})^{\partial \sigma_{2}}$ is a retract of $(\underline{C X}, \underline{X})^{K}$. 
Proof. A new simplicial complex is introduced that will act as an intermediary. In general, a simplicial complex may be characterized by listing its minimal missing faces. Let $\bar{K}$ be the simplicial complex on the vertex set $[m]$ that is characterized by the condition that $M M F(\bar{K})=\left\{\sigma_{1}, \sigma_{2}\right\}$. Intuitively, $\bar{K}$ is obtained from $K$ by filling in all missing faces that do not have either $\sigma_{1}$ or $\sigma_{2}$ as a subface. Rigorously, there is a map of simplicial complexes $K \longrightarrow \bar{K}$ that induces a map of polyhedral products $(\underline{C X}, \underline{X})^{K} \longrightarrow(\underline{C X}, \underline{X})^{\bar{K}}$. Since $\sigma_{1}, \sigma_{2}$ are minimal missing faces of $K$, we have $\sigma_{1}, \sigma_{2} \notin K$ but $\partial \sigma_{1}, \partial \sigma_{2} \subseteq K$. The inclusion $\partial \sigma_{1} \longrightarrow K$ is a map of simplicial complexes and it induces a map of polyhedral products $(\underline{C X}, \underline{X})^{\partial \sigma_{1}} \longrightarrow(\underline{C X}, \underline{X})^{K}$. There is a similar map with respect to $\partial \sigma_{2}$. We will show that the composite $(\underline{C X}, \underline{X})^{\partial \sigma_{1}} \vee(\underline{C X}, \underline{X})^{\partial \sigma_{2}} \longrightarrow(\underline{C X}, \underline{X})^{K} \longrightarrow$ $(\underline{C X}, \underline{X})^{\bar{K}}$ has a left homotopy inverse. Note that this composite of polyhedral products is the same as the one induced by the inclusions $\partial \sigma_{1} \longrightarrow \bar{K}$ and $\partial \sigma_{2} \longrightarrow \bar{K}$, so it suffices to show that the map $(\underline{C X}, \underline{X})^{\partial \sigma_{1}} \vee(\underline{C X}, \underline{X})^{\partial \sigma_{2}} \longrightarrow(\underline{C X}, \underline{X})^{\bar{K}}$ has a left homotopy inverse.

The conditions on the vertex sets $I$ and $J$ imply that $\bar{K}$ has the same form as in Section 3 . Relabelling the spaces $X_{1}, \ldots, X_{m}$ if necessary, we may suppose that the intersection vertex $w$ corresponds to the $m^{t h}$-coordinate space $X_{m}$. By Proposition 2.4, the pushout of simplicial complexes in (1) implies that there is a pushout of polyhedral products

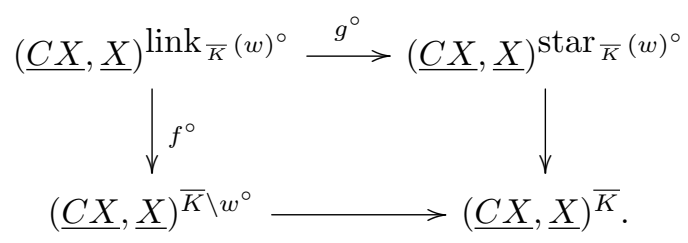

where $\operatorname{link}_{\bar{K}}(w)^{\circ}, \operatorname{star}_{\bar{K}}(w)^{\circ}$ and $\bar{K} \backslash w^{\circ}$ are $\operatorname{link}_{\bar{K}}(w), \operatorname{star}_{\bar{K}}(w)$ and $\bar{K} \backslash w$ regarded as having vertex set $[m]$, and the maps $f^{\circ}$ and $g^{\circ}$ are induced by the inclusions $\operatorname{link} \bar{K}(w)^{\circ} \longrightarrow \bar{K} \backslash w^{\circ}$ and $\operatorname{link}_{\bar{K}}(w)^{\circ} \longrightarrow \operatorname{star}_{\bar{K}}(w)^{\circ}$ respectively. The vertex sets of $\operatorname{link} \bar{K}_{\bar{K}}(w)$ and $\bar{K} \backslash w$ are both $[m-1]$, so by the definition of the polyhedral product,

$$
(\underline{C X}, \underline{X})^{\operatorname{link}_{\bar{K}}(w)^{\circ}}=(\underline{C X}, \underline{X})^{\operatorname{link}_{\bar{K}}(w)} \times X_{m} \quad(\underline{C X}, \underline{X})^{\bar{K} \backslash w^{\circ}}=(\underline{C X}, \underline{X})^{\bar{K} \backslash w} \times X_{m}
$$

and $f^{\circ}=f \times 1$ where $f$ is induced by the inclusion $\operatorname{link} \bar{K}(w) \longrightarrow \bar{K} \backslash w$ and 1 is the identity map on $X_{m}$. On the other hand, the vertex set of $\operatorname{star}_{\bar{K}}(w)$ is $[m]$ so $\operatorname{star}_{\bar{K}}(w)^{\circ}=\operatorname{star}_{\bar{K}}(w)$. Since $\operatorname{star}_{\bar{K}}(w)=\operatorname{link}_{\bar{K}}(w) *\{w\}$, the definition of the polyhedral product implies that

$$
(\underline{C X}, \underline{X})^{\operatorname{star}_{\bar{K}}(w)^{\circ}}=(\underline{C X}, \underline{X})^{\operatorname{link}_{\bar{K}}(w)} \times C X_{m}
$$


and $g^{\circ}=1 \times i_{m}$ where 1 is the identity map on $(\underline{C X}, \underline{X})^{\operatorname{link}_{\bar{K}}(w)}$ and $i_{m}: X_{m} \longrightarrow C X_{m}$ is the inclusion of the base of the cone. Putting all this together, the pushout (2) becomes the pushout

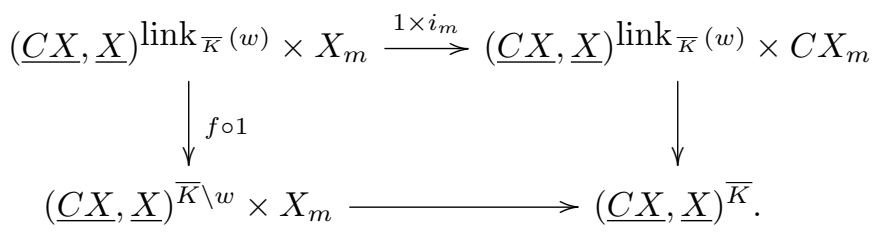

By Lemma $3.3, \bar{K} \backslash w \cong \Delta^{m-2}$, so by Lemma 2.5 (a), $(\underline{C X}, \underline{X})^{\bar{K} \backslash w}=\prod_{i=1}^{m-1} C X_{i}$. Therefore, in (3), both $(\underline{C X}, \underline{X})^{\bar{K} \backslash w}$ and $C X_{m}$ are contractible, implying that (3) is equivalent, up to homotopy, to the homotopy pushout

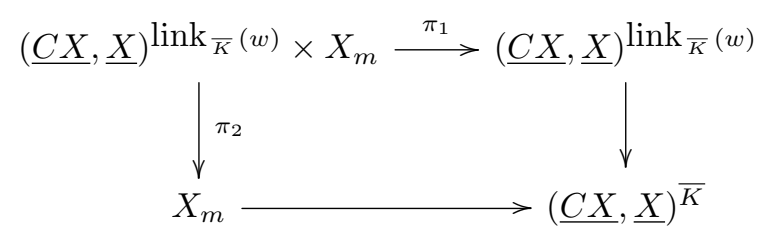

where $\pi_{1}$ and $\pi_{2}$ are the projections onto the first and second factors respectively. It is well known that the pushout of the projections $A \times B \longrightarrow A$ and $A \times B \longrightarrow B$ is homotopy equivalent to the join of $A$ and $B$, which in turn is homotopy equivalent to $\Sigma A \wedge B$. So (4) implies that there is a homotopy equivalence

$$
(\underline{C X}, \underline{X})^{\bar{K}} \simeq \Sigma(\underline{C X}, \underline{X})^{\operatorname{link}_{\bar{K}}(w)} \wedge X_{m}
$$

Now consider the minimal missing faces $\sigma_{1}$ and $\sigma_{2}$ of $\bar{K}$. As in Section 3 , let $\bar{\sigma}_{1}, \bar{\sigma}_{2}$ be the restrictions of $\sigma_{1}, \sigma_{2}$ respectively to the vertex sets $\bar{I}=I \backslash\{w\}, \bar{J}=J \backslash\{w\}$. Note that as $I \neq J$ we also have $\bar{I} \neq \bar{J}$. By Corollary 3.2, $\bar{\sigma}_{1}, \bar{\sigma}_{2} \notin \operatorname{link} \bar{K}_{K}(w)$ but $\partial \bar{\sigma}_{1}, \partial \bar{\sigma}_{2} \subseteq \operatorname{link}_{\bar{K}}(w)$. Therefore, the full subcomplex of $\operatorname{link}_{\bar{K}}(w)$ on $\bar{I}$ is $\partial \bar{\sigma}_{1}$, and the full subcomplex of $\operatorname{link}_{\bar{K}}(w)$ on $\bar{J}$ is $\partial \bar{\sigma}_{2}$. By Proposition 2.2, this implies that $(\underline{C X}, \underline{X})^{\partial \bar{\sigma}_{1}}$ and $(\underline{C X}, \underline{X})^{\partial \bar{\sigma}_{2}}$ are retracts of $(\underline{C X}, \underline{X})^{\operatorname{link}_{\bar{K}}(w)}$. By [2, Theorem 2.21], the fact that $\partial \bar{\sigma}_{1}$ and $\partial \bar{\sigma}_{2}$ are full subcomplexes of $\operatorname{link}_{\bar{K}}(w)$ on different index sets implies that that $\Sigma(\underline{C X}, \underline{X})^{\partial \bar{\sigma}_{1}} \vee \Sigma(\underline{C X}, \underline{X})^{\partial \bar{\sigma}_{2}}$ is a retract of $\Sigma(\underline{C X}, \underline{X})^{\operatorname{link}_{\bar{K}}(w)}$. Thus (5) implies that $\left(\Sigma(\underline{C X}, \underline{X})^{\partial \bar{\sigma}_{1}} \wedge X_{m}\right) \vee\left(\Sigma(\underline{C X}, \underline{X})^{\partial \bar{\sigma}_{2}} \wedge X_{m}\right)$ is a retract of $(\underline{C X}, \underline{X})^{\bar{K}}$.

We wish to choose the retraction more carefully. Restrict $\bar{K}$ to the full subcomplex on the vertex set $I$. Then $M M F\left(\bar{K}_{I}\right)=\left\{\sigma_{1}\right\}$, so $\bar{K}_{I}=\partial \sigma_{1}$. Therefore the star-link-restriction pushout for $\bar{K}_{I}$ with respect to the vertex $w$ becomes

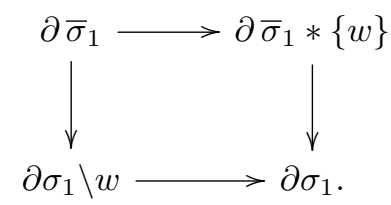

Note that $\partial \sigma_{1} \backslash w$ is the simplex $\Delta^{k-1}$ on the vertex set $\left\{i_{1}, \ldots, i_{k}\right\}$. Now arguing as for $(2)-(4)$ and equation $(5)$, we obtain in place of (5) a homotopy equivalence $(\underline{C X}, \underline{X})^{\bar{K}_{I}}=(\underline{C X}, \underline{X})^{\partial \sigma_{1}} \simeq$ 
$\Sigma(\underline{C X}, \underline{X})^{\partial \bar{\sigma}_{1}} \wedge X_{m}$. Thus we may choose the map $\Sigma(\underline{C X}, \underline{X})^{\partial \bar{\sigma}_{1}} \wedge X_{m} \longrightarrow(\underline{C X}, \underline{X})^{\bar{K}}$ as the composite $\Sigma(\underline{C X}, \underline{X})^{\partial \bar{\sigma}_{1}} \wedge X_{m} \stackrel{\simeq}{\longrightarrow}(\underline{C X}, \underline{X})^{\partial \sigma_{1}} \longrightarrow(\underline{C X}, \underline{X})^{\bar{K}}$. Doing the same for $\partial \sigma_{2}$ we obtain a composite $\left(\Sigma(\underline{C X}, \underline{X})^{\partial \bar{\sigma}_{1}} \wedge X_{m}\right) \vee\left(\Sigma(\underline{C X}, \underline{X})^{\partial \bar{\sigma}_{2}} \wedge X_{m}\right) \stackrel{\simeq}{\longrightarrow}(\underline{C X}, \underline{X})^{\partial \sigma_{1}} \vee(\underline{C X}, \underline{X})^{\partial \sigma_{2}} \longrightarrow$ $(\underline{C X}, \underline{X})^{\bar{K}}$, and it is this composite that has a left homotopy inverse. In particular, we have produced a left homotopy inverse for the map $(\underline{C X}, \underline{X})^{\partial \sigma_{1}} \vee(\underline{C X}, \underline{X})^{\partial \sigma_{2}} \longrightarrow(\underline{C X}, \underline{X})^{\bar{K}}$ induced by the inclusions $\partial \sigma_{1} \longrightarrow \bar{K}$ and $\partial \sigma_{2} \longrightarrow \bar{K}$, as required.

Recall that for $1 \leq i \leq m, Y_{i}$ is the homotopy fibre of the inclusion $A_{i} \longrightarrow X_{i}$.

Theorem 4.2. Let $K$ be a simplicial complex on the vertex set $[m]$ and let $(\underline{X}, \underline{A})$ be any sequence of pointed, path-connected $C W$-pairs. The following hold:

(a) if $M M F(K)=\left\{\sigma_{1}, \ldots, \sigma_{n}\right\}$ and these minimal missing faces are mutually disjoint, then there is a homotopy equivalence

$$
\Omega(\underline{X}, \underline{A})^{K} \simeq\left(\prod_{i=1}^{m} \Omega X_{i}\right) \times\left(\prod_{j=1}^{n} \Omega(\underline{C Y}, \underline{Y})^{\partial \sigma_{j}}\right) ;
$$

(b) if $\sigma_{1}$ and $\sigma_{2}$ are minimal missing faces of $K$ with nontrivial intersection then

$$
\Omega\left((\underline{C Y}, \underline{Y})^{\partial \sigma_{1}} \vee(\underline{C Y}, \underline{Y})^{\partial \sigma_{2}}\right) \text { retracts off } \Omega(\underline{X}, \underline{A})^{K} \text {. }
$$

Proof. By Theorem 2.3, there is a homotopy equivalence

$$
\Omega(\underline{X}, \underline{A})^{K} \simeq\left(\prod_{i=1}^{m} \Omega X_{i}\right) \times \Omega(\underline{C Y}, \underline{Y})^{K}
$$

where, for $1 \leq i \leq m, Y_{i}$ is the homotopy fibre of the inclusion $A_{i} \longrightarrow X_{i}$.

If all of the minimal missing faces of $K$ are mutually disjoint then there is a simplicial isomorphism $K \cong K_{0} * K_{1} * \cdots * K_{n}$ where $K_{0}$ is a product of simplices and, for $1 \leq j \leq n, K_{j}=\partial \sigma_{j}$ (a proof of this may be found in [3], although it may be more commonly known). In general, the definition of a polyhedral product implies that there is a homeomorphism $(\underline{X}, \underline{A})^{L * M} \cong(\underline{X}, \underline{A})^{L} \times(\underline{X}, \underline{A})^{M}$. In our case, as $K_{0}$ is a simplex, Lemma 2.5 (a) implies that $(\underline{C Y}, \underline{Y})^{K_{0}}$ is a product of cones and so is contractible. Thus

$$
(\underline{C Y}, \underline{Y})^{K} \simeq(\underline{C Y}, \underline{Y})^{K_{1}} \times \cdots \times(\underline{C Y}, \underline{Y})^{K_{n}}=(\underline{C Y}, \underline{Y})^{\partial \sigma_{1}} \times \cdots \times(\underline{C Y}, \underline{Y})^{\partial \sigma_{n}} .
$$

Combining this with (6), the homotopy decomposition in part (a) follows.

Next, suppose that $\sigma_{1}$ and $\sigma_{2}$ are minimal missing faces of $K$ that intersect nontrivially. Let $I$ and $J$ be the vertex sets of $\sigma_{1}$ and $\sigma_{2}$ respectively. Let $K_{I \cup J}$ be the full subcomplex of $K$ on the index set $I \cup J$. By Proposition 2.2, $(\underline{C Y}, \underline{Y})^{K_{I \cup J}}$ is a retract of $(\underline{C Y}, \underline{Y})^{K}$. Further, Proposition 4.1 implies that $(\underline{C Y}, \underline{Y})^{\partial \sigma_{1}} \vee(\underline{C Y}, \underline{Y})^{\partial \sigma_{2}}$ is a retract of $(\underline{C Y}, \underline{Y})^{K_{I \cup J}}$. Hence $(\underline{C Y}, \underline{Y})^{\partial \sigma_{1}} \vee(\underline{C Y}, \underline{Y})^{\partial \sigma_{2}}$ is a retract of $(\underline{C Y}, \underline{Y})^{K}$. Combining this with (6), the assertion in part (b) follows.

We now turn to Moore's Conjecture and the distinguishing of elliptic and hyperbolic spaces. For Theorem 1.1, we assume that each pair $\left(X_{i}, A_{i}\right)$ is $\left(D^{n_{i}}, S^{n_{i}-1}\right)$ for $n_{i} \geq 2$. Note that the 
homotopy fibre $Y_{i}$ of the inclusion $S^{n_{i}-1} \longrightarrow D^{n_{i}}$ is also $S^{n_{i}-1}$, so the pair $\left(C Y_{i}, Y_{i}\right)$ in Theorem 4.2 is also homotopy equivalent to $\left(D^{n_{i}}, S^{n_{i}-1}\right)$. Note that as each $X_{i}$ is $D^{n_{i}}$, the term $\prod_{i=1}^{m} \Omega X_{i}$ in Theorem 4.2 (a) is contractible. Also, by Lemma 2.5 (b), each term $(\underline{C Y}, \underline{Y})^{\partial \sigma_{i}}$ in Theorem 4.2 (a) and (b) is homotopy equivalent to a simply-connected sphere.

Proof of Theorem 1.1. Theorem 4.2 (b) implies that if $K$ has two minimal missing faces with nontrivial intersection then a wedge of two simply-connected spheres retracts off $\Omega(\underline{X}, \underline{A})^{K}$. The Hilton-Milnor Theorem shows that a wedge of two such spheres is hyperbolic, and Neisendorfer and Selick [18] showed that a wedge of two such spheres has no exponent at any prime $p$. Hence Moore's conjecture holds in this case. On the other hand, if all the minimal missing faces of $K$ are mutually disjoint then Theorem 4.2 (a) implies that $\Omega(\underline{X}, \underline{A})^{K}$ is homotopy equivalent to a finite product of spheres. This is elliptic, and as each sphere has an exponent at every prime $p$, so does a finite product of them. Hence Moore's Conjecture holds in this case as well.

Proof of Theorem 1.2. Recall that if $Y$ is any space then $\Sigma Y$ is rationally homotopy equivalent to a wedge of spheres. In particular, if $\partial \sigma \subseteq K$ and each $Y_{i}$ is rationally nontrivial then by Lemma 2.5 (b) the space $(\underline{C Y}, \underline{Y})^{\partial \sigma}$ is rationally homotopy equivalent to a wedge of simply-connected spheres. Thus if $v$ is a vertex of $\partial \sigma$ and $\operatorname{rank}\left(\pi_{*}\left(Y_{v}\right) \otimes \mathbb{Q}\right) \geq 2$ then $(\underline{C Y}, \underline{Y})^{\partial \sigma}$ is rationally homotopy equivalent to a wedge of at least two simply-connected spheres.

Suppose that $(\underline{X}, \underline{A})^{K}$ is elliptic. The homotopy decomposition $\Omega(\underline{X}, \underline{A})^{K} \simeq\left(\prod_{i=1}^{m} \Omega X_{i}\right) \times$ $\Omega(\underline{C Y}, \underline{Y})^{K}$ in Theorem 2.3 then immediately implies that each $X_{i}$ must be elliptic, so condition (i) holds. This homotopy decomposition also implies that $(\underline{C Y}, \underline{Y})^{K}$ is elliptic. Let $\sigma_{1}, \ldots, \sigma_{n}$ be the minimal missing faces of $K$. If two of these minimal missing faces intersect, say $\sigma_{1}$ and $\sigma_{2}$, then Theorem 4.2 implies that $\Omega\left((\underline{C Y}, \underline{Y})^{\partial \sigma_{1}} \vee(\underline{C Y}, \underline{Y})^{\partial \sigma_{2}}\right)$ retracts off $\Omega(\underline{C Y}, \underline{Y})^{K}$. Since each of $(\underline{C Y}, \underline{Y})^{\partial \sigma_{1}}$ and $(\underline{C Y}, \underline{Y})^{\partial \sigma_{2}}$ is rationally homotopy equivalent to a wedge of simply-connected spheres, the space $(\underline{C Y}, \underline{Y})^{\partial \sigma_{1}} \vee(\underline{C Y}, \underline{Y})^{\partial \sigma_{2}}$ is rationally homotopy equivalent to a wedge of at least two simplyconnected spheres, implying that it is hyperbolic. Therefore $(\underline{C Y}, \underline{Y})^{K}$ is hyperbolic, a contradiction. Hence the minimal missing faces of $K$ must be mutually disjoint, implying that condition (ii) holds. Because condition (ii) holds, Theorem 4.2 implies that $\Omega(\underline{C Y}, \underline{Y})^{K} \simeq \prod_{j=1}^{n} \Omega(\underline{C Y}, \underline{Y})^{\partial \sigma_{j}}$. It has already been observed that if $v$ is a vertex of $\partial \sigma_{j}$ and $\operatorname{rank}\left(\pi_{*}\left(X_{v}\right) \otimes \mathbb{Q}\right) \geq 2$ then $(\underline{C Y}, \underline{Y})^{\partial \sigma_{j}}$ is rationally homotopy equivalent to a wedge of at least two simply-connected spheres, and so is hyperbolic, implying that $(\underline{C Y}, \underline{Y})^{K}$ is hyperbolic, a contradiction. Thus condition (iii) holds.

Conversely, suppose that conditions (i) to (iii) hold. By Theorem 4.2, condition (ii) implies that $\Omega(\underline{X}, \underline{A})^{K} \simeq\left(\prod_{i=1}^{m} \Omega X_{i}\right) \times\left(\prod_{j=1}^{n} \Omega(\underline{C Y}, \underline{Y})^{\partial \sigma_{j}}\right)$, where $\sigma_{1}, \ldots, \sigma_{n}$ are the minimal missing faces of $K$. For each vertex $v$ of any $\sigma_{i}$, condition (iii) states that $Y_{v}$ is rationally homotopy equivalent to a sphere. Therefore Lemma 2.5 (b) implies that $(\underline{C Y}, \underline{Y})^{\partial \sigma_{i}}$ is rationally homotopy equivalent to a sphere. As each $X_{i}$ is elliptic by condition (i), it has finitely many rational homotopy groups. 
Hence the homotopy decomposition for $\Omega(\underline{X}, \underline{A})^{K}$ implies that $(\underline{X}, \underline{A})^{K}$ has finitely many rational homotopy groups and so is elliptic.

\section{REFERENCES}

1. D.J. Anick, Homotopy exponents for spaces of category two, Algebraic Topology (Arcata, CA, 1986), pp. 24-52, Lecture Notes in Math. 1370, Springer, Berlin, 1989.

2. A. Bahri, M. Bendersky, F.R. Cohen, and S. Gilter, The polyhedral product functor: a method of decomposition for moment-angle complexes, arrangements and related spaces, Adv. Math. 225 (2010), 1634-1668.

3. A. Bahri, M. Bendersky, F.R. Cohen, and S. Gilter, On the rational type of moment-angle complexes, Proc. Steklov Inst. Math. 286 (2014), 219-223.

4. V.M. Buchstaber and T.E. Panov, Toric topology, Mathematical Surveys and Monographs 204, American Mathematical Society, 2015.

5. W. Chachólski, W. Pitsch, J. Scherer and D. Stanley, Homotopy exponents for large H-spaces, Int. Math. Res. Not. IMRN 2008, 16, Art. ID rnn061, 5pp.

6. M.W. Davis and T. Januszkiewicz, Convex polytopes, Coxeter orbifolds and torus actions, Duke Math. J. 62 (1991), 417-452.

7. G. Denham and A. Suciu, Moment-angle complexes, monomial ideals and Massey products, Pure Appl. Math Q. 3 (2007), 25-60.

8. Y. Félix, S. Halperin and J.-C. Thomas, Elliptic spaces II. Enseign. Math. (2) 39 (1993), 25-32.

9. Y. Félix and D. Tanré, Rational homotopy of the polyhedral product functor, Proc. Amer. Math. Soc. 137 (2009), 891-898.

10. J. Grbić and S. Theriault, The homotopy type of the complement of a coordinate subspace arrangement, Topology 46 (2007), 357-396.

11. J. Grbić and S. Theriault, The homotopy type of the polyhedral product for shifted complexes, Adv. Math. $\mathbf{2 4 5}$ (2013), 690-715.

12. K. Iriye and D. Kishimoto, Decompositions of polyhedral products, Adv. Math. 245 (2013), 716-736.

13. I.M. James, The suspension triad of a sphere, Ann. of Math. 63 (1956), 407-429.

14. J. Long, Thesis, Princeton University, 1978.

15. D. McGavran, Adjacent connected sums and torus actions, Trans. Amer. Math. Soc. 251 (1979), $235-254$.

16. C.A. McGibbon and C.W. Wilkerson, Loop spaces of finite complexes at large primes, Proc. Amer. Math. Soc. 96 (1986), 698-702

17. J.A. Neisendorfer, The exponent of a Moore space. Algebraic topology and algebraic K-theory (Princeton, N.J., 1983), 35-71, Ann. of Math. Stud. 113, Princeton Univ. Press, Princeton, NJ, 1987.

18. J. Neisendorfer and P. Selick, Some examples of spaces with and without homotopy exponents, Current trends in algebraic topology, Part 1 (London, Ont., 1981), pp. 343-357, CMS Conf. Proc. 2, Amer. Math. Soc., Providence, R.I., 1982 .

19. G.J. Porter, The homotopy groups of wedges of suspensions, Amer. J. Math. 88 (1966), 655-663.

20. P. Selick, On conjectures of Moore and Serre in the case of torsion-free suspensions, Math. Proc. Cambridge Philos. Soc. 94 (1983), 53-60.

21. M. Stelzer, Hyperbolic spaces at large primes and a conjecture of Moore, Topology 43 (2004), 667-675.

22. H. Toda, On the double suspension $E^{2}$, J. Inst. Polytech. Osaka City Univ., Ser. A 7 (1956), 103-145. 
School of Mathematical Sciences and LPMC, Nankai University, Tianjin 300071, P.R. China E-mail address: haoyanlong13@mail.nankai.edu.cn

School of Mathematical Sciences and LPMC, Nankai University, Tianjin 300071, P.R. China E-mail address: qwsun13@mail.nankai.edu.cn

Mathematical Sciences, University of Southampton, Southampton SO17 1BJ, United Kingdom E-mail address: S.D.Theriault@soton.ac.uk 